\title{
Integrating hydrometeorological information for rainfall-runoff modelling by artificial neural networks
}

\author{
Yen-Ming Chiang and Fi-John Chang* \\ Department of Bioenvironmental Systems Engineering National Taiwan University No. 1, Sec. 4, Roosevelt Road Taipei 10617 Taiwan (R.O.C.)
}

\begin{abstract}
:
The major purpose of this study is to effectively construct artificial neural networks-based multistep ahead flood forecasting by using hydrometeorological and numerical weather prediction (NWP) information. To achieve this goal, we first compare three mean areal precipitation forecasts: radar/NWP multisource-derived forecasts $\left(P_{r}\right)$, NWP precipitation forecasts $\left(P_{n}\right)$, and improved precipitation forecasts $\left(P_{m}\right)$ by merging $P_{r}$ and $P_{n}$. The analysis shows that the accuracy of $P_{m}$ is higher than that of $P_{r}$ and $P_{n}$. The analysis also indicates that the NWP precipitation forecasts do provide relative effectiveness to the merging procedure, particularly for forecast lead time of 4-6 h. In sum, the merged products performed well and captured the main tendency of rainfall pattern. Subsequently, a recurrent neural network (RNN)-based multistep ahead flood forecasting techniques is produced by feeding in the merged precipitation. The evaluation of $1-6$-h flood forecasting schemes strongly shows that the proposed hydrological model provides accurate and stable flood forecasts in comparison with a conventional case, and significantly improves the peak flow forecasts and the time-lag problem. An important finding is the hydrologic model responses which do not seem to be sensitive to precipitation predictions in lead times of $1-3 \mathrm{~h}$, whereas the runoff forecasts are highly dependent on predicted precipitation information for longer lead times (4-6 h). Overall, the results demonstrate that accurate and consistent multistep ahead flood forecasting can be obtained by integrating predicted precipitation information into ANNs modelling. Copyright (c) 2009 John Wiley \& Sons, Ltd.
\end{abstract}

KEY WORDS artificial neural networks; multistep ahead of flood forecasting; numerical weather prediction; radar; quantitative precipitation forecasting

Received 2 September 2008; Accepted 9 February 2009

\section{INTRODUCTION}

Precipitation is perhaps one of the most important weather elements in the hydrological cycle. In its liquid form it can be annoying when light, disruptive of a wide range of activities when moderate, and fatally destructive when intense. Yet, its absence can ultimately lead to even greater loss of life (Golding, 2000). The water resources in Taiwan are highly dependent on torrential rain and typhoons which are typically associated with heavy rainfall. The average annual rainfall is about $2500 \mathrm{~mm}$, while typhoon-related rainfall has been recorded at over $1000 \mathrm{~mm} /$ day. Consequently, prediction of the intensity of precipitation is recognized as the most important study for watershed management and flood mitigation in Taiwan. The reliability and accuracy of short-term flood forecasting will be effectively increased if precise prediction of precipitation can be provided. For this reason, efforts have been focused on improving the accuracy of rainfall prediction using different data (Grecu and Krajewski, 2000; Kidd et al., 2003; Mittermaier, 2008) and techniques (Minns and Hall, 1996; Yussouf and Stensrud, 2008).

\footnotetext{
* Correspondence to: Fi-John Chang, Department of Bioenvironmental Systems Engineering, National Taiwan University, No. 1, Sec. 4, Roosevelt Road, Taipei 10617, Taiwan (R.O.C). E-mail: changfj@ntu.edu.tw
}

Various hydrological models have been proposed for modelling runoff/flood forecasts. One of the most popular_methods involves artificial neural networks (ANNs) which are often used for finding rainfall-runoff characteristics (Luk et al., 2000; Giustolisi and Laucelli, 2005; Chang and Chang, 2006; Chaves and Kojiri, 2007; Chiang et al., 2007b; Firat and Güngör, 2008). ANNs are complex information processing systems that have been shown to act as function approximations. The major advantage of using ANNs is that they are effective in extracting significant features from complex data structure. Furthermore, ANNs are capable of learning and simulating any relationship between a series of inputs and outputs if sufficient amount and complexity of training data are provided. For their abilities in simplifying calculation and enhancing the adaptability, ANNs have been successfully used in various problems that are difficult to understand, and have been proven to be an efficient method to traditional methods for hydrological modelling (Hsu, et al., 1995; Sivakumar et al., 2002; Chang et al., 2004).

In rainfall-runoff simulation, the hydrological model would be more beneficial if longer forecast lead times could be provided, in terms of time for damage reduction. Thus, multistep ahead of forecast is crucial for water resource management and flood warning. It is well known that the accuracy of hydrologic responses depends heavily on the effectiveness of precipitation information. 
However, ground-based gauge measurements are usually incapable of providing useful information with sufficient lead time for this purpose. Owing to the lack of real data in the future, precise and successful multistep ahead of flood forecasting proves to be a great challenge (Chang et al., 2007). Therefore, accurate and reliable quantitative precipitation forecasting (QPF) information is undoubtedly required for precise multistep ahead of flood forecasting, because it is the most important input for a hydrological model.

QPF has provided useful and essential information for practical applications in various fields such as reservoir operation, water resources management, agriculture, and flood monitoring. With the availability and feasibility of measurements and technologies, QPF has been continuously developed in the past few years. However, the improvement in precipitation prediction is somewhat limited due to the complex interactions within the precipitation processes. Several factors contribute to the difficulties in forecasting the precipitation. Precipitation can occur over large scales, varying from large air mass movements to extremely localized convective events (Rogers and Yau, 1989). Precipitation is further influenced by air motion and turbulent eddies, aerosol properties, and microphysical processes that dictate droplet growth and evaporation (Ganguly and Bras, 2003). Simply stated, the precipitation rate varies extremely in time and space, and it is impossible even to precisely define the edge of a rain area. Traditional methods used for QPF remain extrapolation techniques that simply assume that rainfall intensities at forecast lead times are identical to the most recent measurements. Nevertheless, spatial variability of precipitation can translate into significant variations of simulated runoff peaks and phases.

The major purpose of this study is to provide precise, stable, and practical multistep ahead of flood forecasts. We first compare three 1-6-h QPF products, namely radar/NWP multisource-derived precipitation forecasts, NWP precipitation forecasts, and merged precipitation forecasts. In order to obtain more accurate QPF, a preliminary study on investigating the potential and the effectiveness of merging two forecasted rainfall sources is presented. Second, the recurrent neural networks (RNNs) are constructed for 1-6-h flood forecasting by using the QPF information as model input. The organization of this paper is as follows. The next section presents the study area and hydrometeorological data including NWP outputs, streamflow, precipitation, and radar observations. Description of an ANN-based QPF model, the precipitation-merged method, as well as the RNN-based hydrological model is provided in Section 3. Section 4 provides a comparison of different QPF products and an evaluation of forecasted hydrologic responses. Finally, the conclusions are given in Section 5.

\section{STUDY AREA AND DATA}

\section{Study area}

The Wu-Tu watershed was selected as the study area where the Keelung River wanders through the watershed. Located in northern Taiwan, the Keelung River is one of the major tributaries of the Tanshui River which surrounds the metropolis of Taipei. Locations of the watershed and radar station are shown in Figure 1. The insert shows an enlargement of $\mathrm{Wu}-\mathrm{Tu}$ watershed, where the streamflow gauge is denoted as $S_{1}$ and three rain gauges are denoted as $R_{1}, R_{2}$, and $R_{3}$. Owing to the rugged topography of the watershed, flash floods arrive in the middle and downstream rapidly (around 2-6 h) and can cause great damage. It is imperative to construct an accurate multistep ahead of flood forecasts in this watershed in order to provide information for the design and planning of water resources management and for the determination of flood risk.

\section{Gauge measurements}

The outlet of the watershed is at Wu-Tu station $\left(\mathrm{S}_{1}\right)$, where hourly runoff $\left(\mathrm{m}^{3} / \mathrm{s}\right)$ measurements were collected from eight typhoon events within the time period of 2004 and 2005. For the same observational periods, precipitation $(\mathrm{mm})$ with a recording resolution of $1 \mathrm{~h}$ at these gauges was also collected. For the multistep ahead of forecasting of floods, the input of the hydrological model was fed with mean areal precipitation. Thus, the represented precipitation from the above-mentioned rain gauges is estimated to be the mean areal precipitation $\left(P_{g}\right)$ by using the Thiessen method. Table 1 shows the configuration of eight typhoon events in different phases, number of data, maximum precipitation, and total precipitation of every event. Four typhoon events were arranged in the training set and both validation and testing

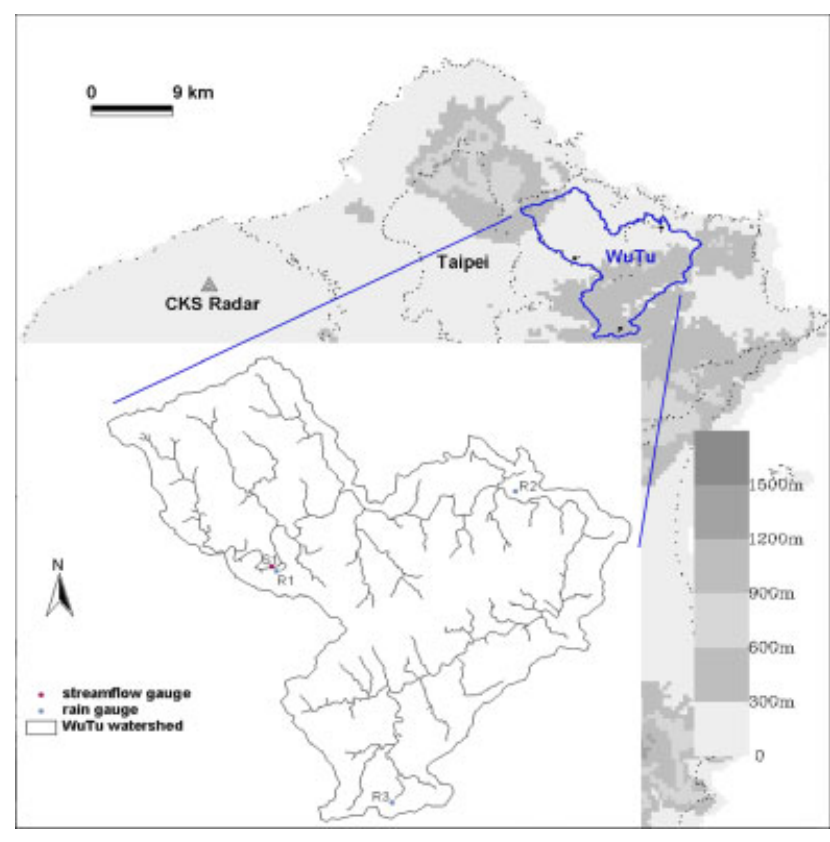

Figure 1. Map of study area with positions of gauge and radar stations 
Table I. The selected typhoon events and their configuration

\begin{tabular}{lllrcc}
\hline Event & Configuration & Name & $\begin{array}{r}\text { \# of } \\
\text { data }\end{array}$ & $\begin{array}{c}\text { Max } \\
(\mathrm{mm} / \mathrm{hr})\end{array}$ & $\begin{array}{c}\text { Total } \\
(\mathrm{mm})\end{array}$ \\
\hline 1 & Training & RANANIM & 96 & 19 & 135 \\
2 & & AERE & 120 & 31 & 344 \\
3 & & HAIMA & 120 & 45 & 511 \\
4 & & MINDULLE & 96 & 13 & 177 \\
5 & \multirow{2}{*}{ Validation } & NANMADOL & 72 & 51 & 299 \\
6 & & HAITANG & 120 & 25 & 268 \\
7 & \multirow{2}{*}{ Testing } & NOCKTEN & 120 & 53 & 438 \\
8 & & MATSA & 96 & 31 & 261 \\
\hline
\end{tabular}

sets consisted of two events. The training sets are used to adjust the connected weights of the constructed networks, and the validation sets are implemented to determine the epoch at which training should be stopped to avoid overfitting (Chiang et al., 2004). If the validation sets indicate that the network is overfitting, then the network should be retrained using a different set of parameters. Finally, the reliability of the selected network is evaluated by using the testing sets.

\section{Radar observations}

The radar observations used to construct precipitation forecasts herein are taken from the Doppler radar at Chiang Kai-Shek (CKS) station (see Figure 1) that has an effective range of $120 \mathrm{~km}$ with Doppler mode. The radar parameter of interest to this research is the reflectivity factor at horizontal polarization and is recorded once every $\mathrm{km}$ for each azimuthal scan. Detailed analysis and information about the radar and data pre-process can be found in Chiang et al. (2007a). After processing, the grid size in the study area is approximately $1 \times$ $1 \mathrm{~km}$, and the temporal resolution is $1 \mathrm{~h}$. The hourly constant altitude plan position indicators (CAPPI) with the same observational periods as gauge measurements are generated at different altitudes.

\section{NWP model outputs}

The NWP output products used in this study have been generated by a regional non-hydrostatic forecast system (NFS) model operated at the central weather bureau (CWB), Taiwan. The NFS model generates forecasts for precipitation and other meteorological state variables within the $5^{\circ} \mathrm{S}-43^{\circ} \mathrm{N}$ and $78^{\circ} \mathrm{E}-180^{\circ} \mathrm{E}$ region with a spatial resolution of $45 \mathrm{~km}$. The NFS model runs four times daily $(0000,0600,1200$, and 1800 UTC) and a three-hourly forecast cycle is produced up to $72 \mathrm{~h}$ for each operation. The output products of the NFS consisted of fifteen atmospheric variables which partially included sixteen vertical levels. Although the meteorological state variables are expected to contribute useful information about the precipitation pattern to the ANN (Grimes et al., 2003), it is impossible to calibrate the network model with all the NWP outputs since this will require a huge amount of computer resources and training time. Consequently, the number of predictors of the
NFS model should be reduced. The ability of the NWP models, however, is not the main purpose of this study, hence it will not be discussed here. Since the most important large-scale factor in the production of precipitation is moisture, the following variables are frequently suggested in the literature (Kuligowski and Barros, 1998) and, therefore, were directly extracted from the NFS output fields.

For the moisture factors, the precipitation forecasts $\left(P_{n}\right)$ and four of the relative humidity variables at levels of $1000,925,850$, and $700 \mathrm{hPa}\left(R H_{1000}, R H_{925}\right.$, $R H_{850}$, and $R H_{700}$ ) were taken from NFS outputs. It is important to note that the number of levels used for determining variables may vary with problems and geographical location. For example, in Kuligowski and Barros's work (1998), the select meteorological variables were extracted from 1000 to $500 \mathrm{hPa}$ levels, whereas the $700 \mathrm{hPa}$ humidity and the $850 \mathrm{hPa}$ air temperature are used for rainfall forecasting (Valverde Ramirez et al., 2005). The selection of NWP output level in this study was determined according to the vertical altitude of the 3-D reflectivities structure. Basically, the altitude of data at $700 \mathrm{hPa}$ level is about $3000 \mathrm{~m}$ which covers the 3-D reflectivity structure used in this study. Other model variables that were extracted from the NFS outputs included pressure (PRES), temperature (TMP), and pressure vertical velocity $(V V E L)$. The latter two variables were obtained for the same four levels as relative humidity.

Besides, we found the correlation coefficients between the meteorological variables (i.e. relative moisture, temperature and vertical velocity) and precipitation are small (less than 0.35) indicating that all these NWP meteorological variables have low linear correlation with precipitation. Nevertheless, it is difficult to exactly trim the input variables since the relation between these meteorological variables and precipitation could be either linear or nonlinear. Furthermore, the ANNs are capable of learning any relationship between a series of input and output variables and can extract the significant features inside the data structure. From the above reasons, the trimming of the NWP variables will not be considered in the present study. Overall, there will be thirteen meteorological state variables and one precipitation variable extracted from the NFS outputs. Before applying the predictor set of NFS model for further application, it is necessary to scale the time of the NFS outputs in accordance with that of rain gauges. To achieve forecasts on an hourly scale, most of the previous works (Sokol, 2006) use the linear interpolation technique. Accordingly, linear interpolation was introduced to obtain the required hourly series. In other words, the differences of data scales among various sources were transferred to uniform scale to facilitate the following applications.

\section{APPLICATION}

\section{Description of a BPNN-based QPF model}

There have been many attempts in the development ofQPF, some based on numerical models and some 
on nowcasting techniques. On the modelling aspect, although the formulations and physical processes are becoming more and more sophisticated, the accuracy of QPF is still rather low when the forecast target involves severe precipitation events of short duration, e.g. typhoons. Nonetheless, it is recognized that model simulations could provide very useful guidance about the development of convective systems (Droegemeier et al., 2000). Therefore, an ANN-based model was built for a 1-6 h QPF by considering the gauge measurements, meteorological variables of NFS output fields, and a 3-D structure of radar echo. A three-layer back-propagation neural network (BPNN) with conjugate gradient algorithm (Chiang et al., 2004) was utilized for radar/NWP multisource-derived 1-6-h precipitation forecasting $\left(P_{r}\right)$.

It should be noted that the selection of learning algorithm for model training is basically problem-dependent and somewhat a trade-off. For example, the steepest descent method often yields a local optimal solution even if the searching speed of the method is fast. The conjugate gradient method, however, represents a good compromise between simplicity of the steepest descent method and the fast quadratic convergence of Newton's method (Ham and Kostanic, 2001) and has now become more popular for model calibration. In practice, the process makes good uniform progress toward the solution at every time step and has been found to be effective in finding a better solution than the steepest descent method (Chiang et al., 2004; Kisi, 2007) and the Levenberg-Marquardt learning method (Chattopadhyay and Chattopadhyay, 2008), and therefore, is widely used for training the neural networks (Khan and Coulibaly, 2006).

The size of the 3-D reflectivity factor structure was determined by the horizontal advection and vertical (terminal) velocity of raindrops. In other words, the size of the data grid is enlarged as altitudes increase. Detailed information on the determination of the 3-D reflectivity structure can be found in Chiang et al. (2007a). In sum, the sizes of CAPPI grid at four different elevations are $15 \times 15 \mathrm{~km}, 11 \times 11 \mathrm{~km}, 7 \times 7 \mathrm{~km}$, and $3 \times 3 \mathrm{~km}$ (see Figure 2). Moreover, in order to efficiently reduce the input dimensions of the BPNN model, four spatial mean

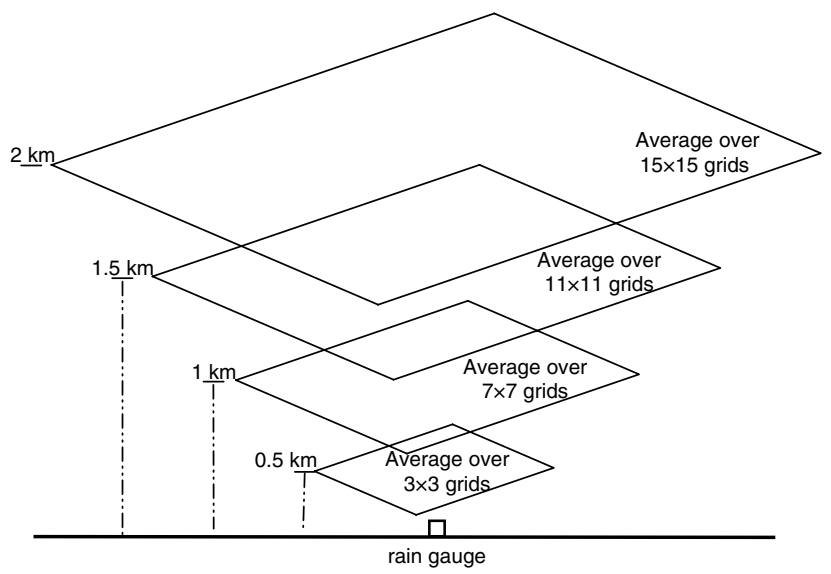

Figure 2. Three-dimensional structure of radar echo for ANN inputs reflectivities averaged over these four elevations (Xiao and Chandrasekar, 1997) were adopted in this study. The 3-D reflectivity structures were applied to BPNN as the inputs and the corresponding mean areal precipitation data $\left(P_{g}\right)$ were used as the target output. There are six single-output BPNNs built for a 1-6-h precipitation forecasting. The upper right corner of Figure 3 illustrates the BPNN-based QPF models that take into account three sources: gauge measurement, NWP meteorological variables, and radar observations. Overall, the input dimensions of BPNN are 28, consisting of 13 NFS meteorological predictors, 12 spatial mean reflectivities, and 3 previous rainfall values $\left(P_{g}(t), P_{g}(t-1)\right.$, and $\left.P_{g}(t-2)\right)$.

\section{QPF by merging radar-derived and NWP-derived precipitation forecasts}

Accurate short-term $(1-6 \mathrm{~h})$ precipitation predictions are important for a precise multistep ahead of flood forecasting in the watersheds of Taiwan. Traditionally, numerical weather prediction models can provide such information. However, of the NWP output fields, QPF is usually the one with the highest variability in space and time, and is thought to be least accurate. In general, precipitation forecasting from the NWP models is an average over grid-point values, therefore being a function of the model spatial resolution. This is rather inadequate for rainfall, which is highly variable both in space and time. Nevertheless, some recent studies have indicated that using hybrid approaches could have potential contribution to precipitation forecasting by statistically combining QPF from NWP and extrapolation (Ganguly and Bras, 2003; Sokol, 2006). Golding (2000) also mentioned that improving QPF at 1-6-h lead time is a key requirement, and this could be achieved by statistically combining radar and NWP information.

Therefore, an improved QPF product merged from radar/NWP multisource-derived forecasts $\left(P_{r}\right)$ and NWP precipitation forecasts $\left(P_{n}\right)$ is presented to generate 1-6-h precipitation forecasting during typhoon periods. Figure 3 shows a schematic flowchart for the proposed

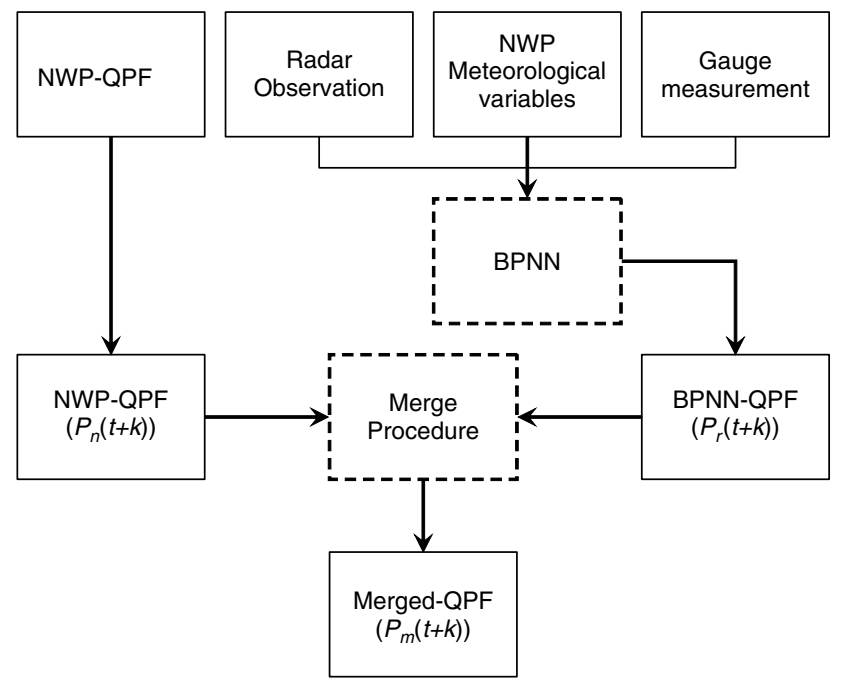

Figure 3. A schematic flowchart for the merged QPF 
merging strategy. The final product of the proposed approach is the merged QPF $\left(P_{m}\right)$ which is composed of NWP-QPF and BPNN-QPF. Case studies were made of the merged model with lead time $k$ varying from 1 to $6 \mathrm{~h}$. The study first evaluates the accuracy of the merged QPF and then investigates the effectiveness and potential improvement of merged products on a 1-6-h flood forecasting. The procedure of merging two QPF products is calculated as follows:

$$
P_{m}(t+k)=P_{r}(t+k) \times \theta_{k}+P_{n}(t+k) \times\left(1-\theta_{k}\right)
$$

where $0 \leq \theta_{k} \leq 1$; the $P_{m}(t+k)$ represents the merged precipitation with lead time $k$, and the $\theta_{k}$ means the merging weighting factor for the radar-derived forecasts. The $P_{r}(t+k)$ and $P_{n}(t+k)$ represent the mean areal precipitation derived from radar and NWP forecasts, respectively.

\section{RNN-based hydrological models}

For merging the precipitation forecasts to 1-6-h flood forecasting, two modules were included: (1) a precipitation-merged model, and (2) a basin-scale hydrological model. The precipitation-merged model is set to a linear model, while the hydrological model is implemented by a RNN as shown in Figure 4. The merging weighting factor was optimized via minimizing the hydrological forecast error by using grid-based searching, whereas the network parameters were trained by a gradient method (Ham and Kostanic, 2001). The architecture of the RNN consists of an input layer, a hidden layer, and an output layer. Both hidden and output layers have internal time-delay feedback loops which are fully connected.

The inputs of the network consist of the precipitation and runoff information; three cases of optional precipitation inputs are considered: (1) historical gauge measurements up to the present, (2) gauge measurements used as predicted precipitation, and (3) merged precipitation forecasts. Case 1 only uses the historical (past) gauge records for model training. No predicted variables are used. This is the common situation where hydrologists rely on past observations to predict future values. Case 2 uses gauge measurements as the predicted precipitation. This is an ideal situation, given that perfect precipitation predictions do not exist. Case 3 combines two predicted precipitation sources to generate the merged product and then is used for 1-6-h flood forecasting. For Cases 1 and 2,

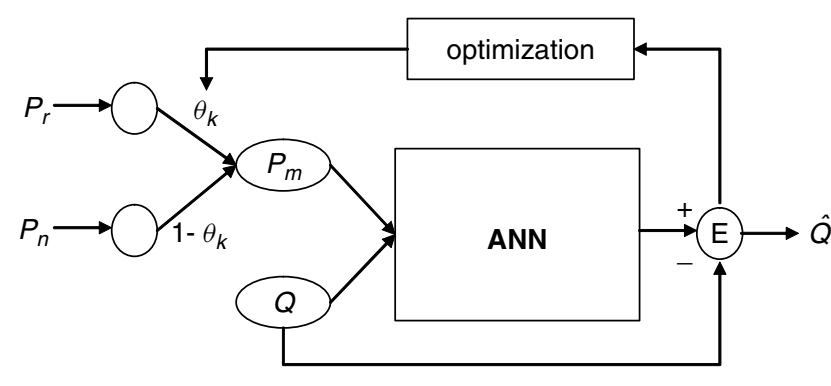

Figure 4. The architecture of a neural network-based merging module there is no precipitation-merging module included in the $\mathrm{RNN}$, and the $P_{m}$ is directly replaced by gauge values. The only difference is that the precipitation information is kept at the current time $t\left(P_{g}(t)\right)$ in Case 1 no matter what forecasted lead time is; whereas the precipitation information varies with the lead time $\left(P_{g}(t+k)\right)$ in Case 2. For Case 3 , the merging parameter, $\theta_{k}$, is in the range of $0-1$. When merging from two separate sources, the RNN takes the combined precipitation as its input. Hydrologic responses from a precipitation sequence are generated from the RNN models. The RNN output is the runoff prediction of the watershed. The RNN model is denoted as $\mathrm{RNN}_{\mathrm{G}}$ and $\mathrm{RNN}_{\mathrm{I}}$ when the precipitation input is gauge and ideal value, respectively. For the merged precipitation product, the RNN model was assigned as $\mathrm{RNN}_{\mathrm{M}}$. With various settings of precipitation inputs provided, the performance of RNN models in flood forecasting is evaluated.

To investigate the stability and generalizing capability of the constructed networks based on different sources of input information, the RNNs were trained from the individual input sources, as for two $P_{g}$ sequences (conventional and ideal) and $P_{m}$ separately. Because every RNN was trained by an individual rainfall sequence, the calibration procedure gives the best RNN fitted weights for the assigned rainfall source for those training events. In a part of the search process, through a trial and error procedure, the number of hidden neurons was set to three. Detailed input variables of these RNN models are displayed in Table 2.

\section{Statistics for model assessment}

Several statistical criteria were selected for the evaluation of QPFs and multistep ahead of flood forecasting. The agreement between observations and predictions was estimated based on correlation coefficient (CC), root mean square error (RMSE), coefficient of efficiency (CE), and mean absolute error (MAE). The first two criteria were used to evaluate the performance of several precipitation forecasts. The CC and CE indices are widely used in hydrology to estimate the efficiency of the fit, and hence, facilitate comparison of the study results. The RMSE is used as a common performance measure because it weights heavily the larger errors. The MAE, however, computes all deviations from the original data and is not weighted towards high values.

Table II. Input variables of RNN models with lead times vary from 1 to $6 \mathrm{~h}$

\begin{tabular}{lccc}
\hline Lead time & \multicolumn{3}{c}{ Model input variables } \\
\cline { 2 - 4 } & $\mathrm{RNN}_{\mathrm{G}}$ & $\mathrm{RNN}_{\mathrm{I}}$ & $\mathrm{RNN}_{\mathrm{M}}$ \\
\hline 1 & $Q(t), P_{g}(t)$ & $Q(t), P_{g}(t+1)$ & $Q(t), P_{m}(t+1)$ \\
2 & & $Q(t), P_{g}(t+2)$ & $Q(t), P_{m}(t+2)$ \\
3 & & $Q(t), P_{g}(t+3)$ & $Q(t), P_{m}(t+3)$ \\
4 & & $Q(t), P_{g}(t+4)$ & $Q(t), P_{m}(t+4)$ \\
5 & & $Q(t), P_{g}(t+5)$ & $Q(t), P_{m}(t+5)$ \\
6 & & $Q(t), P_{g}(t+6)$ & $Q(t), P_{m}(t+6)$ \\
\hline
\end{tabular}


For a flood forecasting scheme, accurately predicting the timing and the value of peak flow would be a great benefit to the management of flood warning and mitigation. Therefore, the forecasted features of interest are evaluated by two criteria: the error in the timing of a predicted peak discharge $\left(E T_{p}\right)$ and percent error in modelled peak discharge relative to observed flow $\left(E Q_{p}\right)$. These two statistical indices are defined as follows.

$$
\begin{aligned}
& E T_{p}=\hat{T}_{p}-T_{p} \\
& E Q_{p}=\frac{\hat{Q}_{p}(i)-Q_{p}(i)}{Q_{p}(i)} \times 100 \%
\end{aligned}
$$

where $\hat{T}_{p}$ is the timing of the predicted peak discharge to arrive, $T_{p}$ is the time required for the observed peak discharge to arrive; $\hat{Q}_{p}$ and $Q_{p}$ are the peak values of forecasted and observed flood, respectively.

In addition, a skill score (SS) was also used for evaluating the percentage improvement in any target model with respect to the forecasts of a reference model. This statistic is given by:

$$
S S=\left(\frac{E_{R M}-E_{T M}}{E_{R M}}\right) \times 100 \%
$$

where $E_{T M}$ is the statistical error measurement in any target model and $E_{R M}$ is the statistical error measurement of a reference model. The values of skill score were computed from RMSE statistics in this specific case. A positive $S S$ indicates the performance of target model is better than that of the reference model. In this experiment, we further divided skill score into two types: $S S_{1}$ and $S S_{2}$ for QPF and multistep ahead of flood forecasting, respectively. For $S S_{1}$, the $E_{R M}$ is computed from $P_{g}$ and $E_{T M}$ is gained from $P_{n}, P_{r}$, or $P_{m}$; whereas the $E_{R M}$ is calculated from $R N N_{G}$ and the $E_{T M}$ is obtained from $R N N_{M}$ forecasts for $S_{2}$.

\section{RESULTS}

\section{Results of $Q P F s$}

Table 3 summarizes the comparative results of the above three methods as compared with $P_{g}$ for 1-6-h precipitation forecasts. First, when forecast lead times are less than $3 \mathrm{~h}$ it appears that $P_{r}$ produce much better performance in terms of higher $\mathrm{CC}$ and smaller

\begin{tabular}{|c|c|c|c|c|c|c|}
\hline \multirow[t]{2}{*}{ Lead time } & \multicolumn{2}{|c|}{$P_{n}$} & \multicolumn{2}{|c|}{$P_{r}$} & \multicolumn{2}{|c|}{$P_{m}$} \\
\hline & $\mathrm{CC}$ & RMSE & $\mathrm{CC}$ & RMSE & $\mathrm{CC}$ & RMSE \\
\hline 1 & 0.42 & 6.89 & 0.71 & 5.66 & 0.72 & $5 \cdot 61$ \\
\hline 2 & 0.41 & 6.85 & 0.58 & 6.5 & 0.61 & $6 \cdot 31$ \\
\hline 3 & 0.41 & $7 \cdot 10$ & 0.53 & 6.97 & 0.60 & 6.40 \\
\hline 4 & 0.41 & $7 \cdot 15$ & 0.44 & 7.40 & 0.55 & 6.75 \\
\hline 5 & 0.40 & $7 \cdot 51$ & 0.37 & 7.93 & 0.50 & $7 \cdot 08$ \\
\hline 6 & 0.37 & 8.03 & $0 \cdot 32$ & 8.93 & 0.44 & 7.75 \\
\hline
\end{tabular}
RMSE, than those obtained from $P_{n}$. For four-hour-ahead

Table III. Statistics of three forecasted precipitation products precipitation forecasts, the RMSE computed from $P_{n}$ becomes slightly smaller than that of $P_{r}$, but the CC value of $P_{n}$ is still a little lower than that of $P_{r}$. The inconsistency of these two statistics between $P_{n}$ and $P_{r}$ can be explained by the fact that $P_{r}$ forecasts result in larger bias during heavy rainfall periods (worse RMSE) even though the major trend of the precipitation has been captured by $P_{r}$ (better $\mathrm{CC}$ ). On the other hand, both statistics indicate that $P_{n}$ has better accuracy of forecasts than that of $P_{r}$ for forecast lead time greater than $5 \mathrm{~h}$.

Secondly, from the aspect of $P_{n}$ forecasts, the CC index is quite similar $(0.42 \sim 0.37)$ for forecast lead time varying from 1 to $6 \mathrm{~h}$, and the value of RMSE only changes from 6.89 to 8.03 ; whereas the accuracy of $P_{r}$ sequence is reduced as the forecast lead time is increasing even if the $P_{r}$ sequence produces an excellent one-hour-ahead prediction. These results indicate that the precipitation forecasts generated from the NWP model are considerably stable, although the precision of forecasts for the first few lead times is relatively low. Nevertheless, the results are consistent with the characteristic of NWP models that numerical models usually produce less accuracy of forecasts at the beginning steps due to the turbulence caused by inappropriate initial conditions (Meneguzzo et al., 2004). Finally, an inspection of the merged precipitation products clearly shows that the $P_{m}$ sequence has the best performance of the 1-6$\mathrm{h}$ precipitation forecasts among all methods. For each forecast lead time, the merged model seems to obtain potential information and/or benefit from both sources, and therefore, generates improved precipitation forecasts. The performance of the $P_{m}$ not only has the advantages of both sequences but improves the accuracy for 1-6-h precipitation forecasts. In other words, the $P_{m}$ contains the characteristic of high accuracy for 1-3-h forecasts, and maintains the stability of prediction for lead time greater than $4 \mathrm{~h}$.

Figure 5 illustrates the variation of $\mathrm{CC}$ from three QPF sequences for lead time ranging from 1 to $6 \mathrm{~h}$. It should be noticed that the red line displayed in Figure 5 represents the autocorrelation of ground-based gauge rainfall, $P_{g}$, in 1 to $6 \mathrm{~h}$ lead time. It is amazing that the autocorrelation of $P_{g}$ rapidly decreased (from 0.74 to 0.17 ) as forecast lead time increased. However, it is interesting to note that the CC index of $P_{g}$ is superior to all methods for one-hour-ahead prediction. This may be due to the rainfall pattern of a typhoon being concentrated within a specific period; therefore resulting in a high correlation between gauge measurements at time $t$ and $t+1$. Inspection of this figure clearly indicates the CC value of $P_{g}$ is smaller than both $P_{r}$ and $P_{m}$ when lead time is greater than $1 \mathrm{~h}$, and the accuracy of $P_{g}$ is even worse than that of $P_{n}$ from a lead time of $3 \mathrm{~h}$. The results also demonstrate that all model-based forecasts provide better information than gauge measurements, especially for lead time greater than $2 \mathrm{~h}$. Figure 6(a) shows the rainfall pattern obtained from gauge measurements in testing phase and Figure 6(b)-(d) display the forecasted 


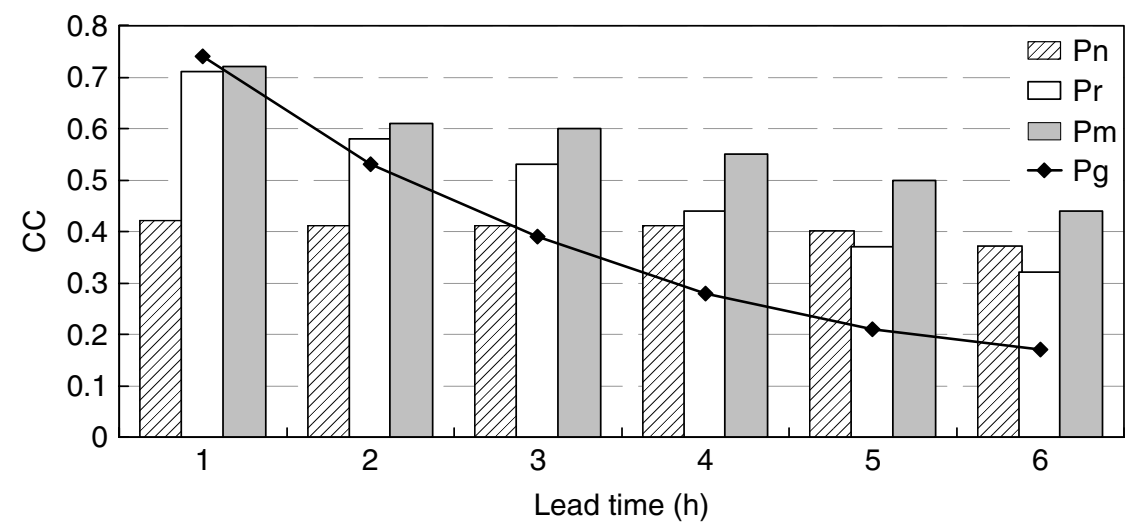

Figure 5. The variations of $\mathrm{CC}$ values obtained from various precipitation products for $1-6-\mathrm{h}$ lead time
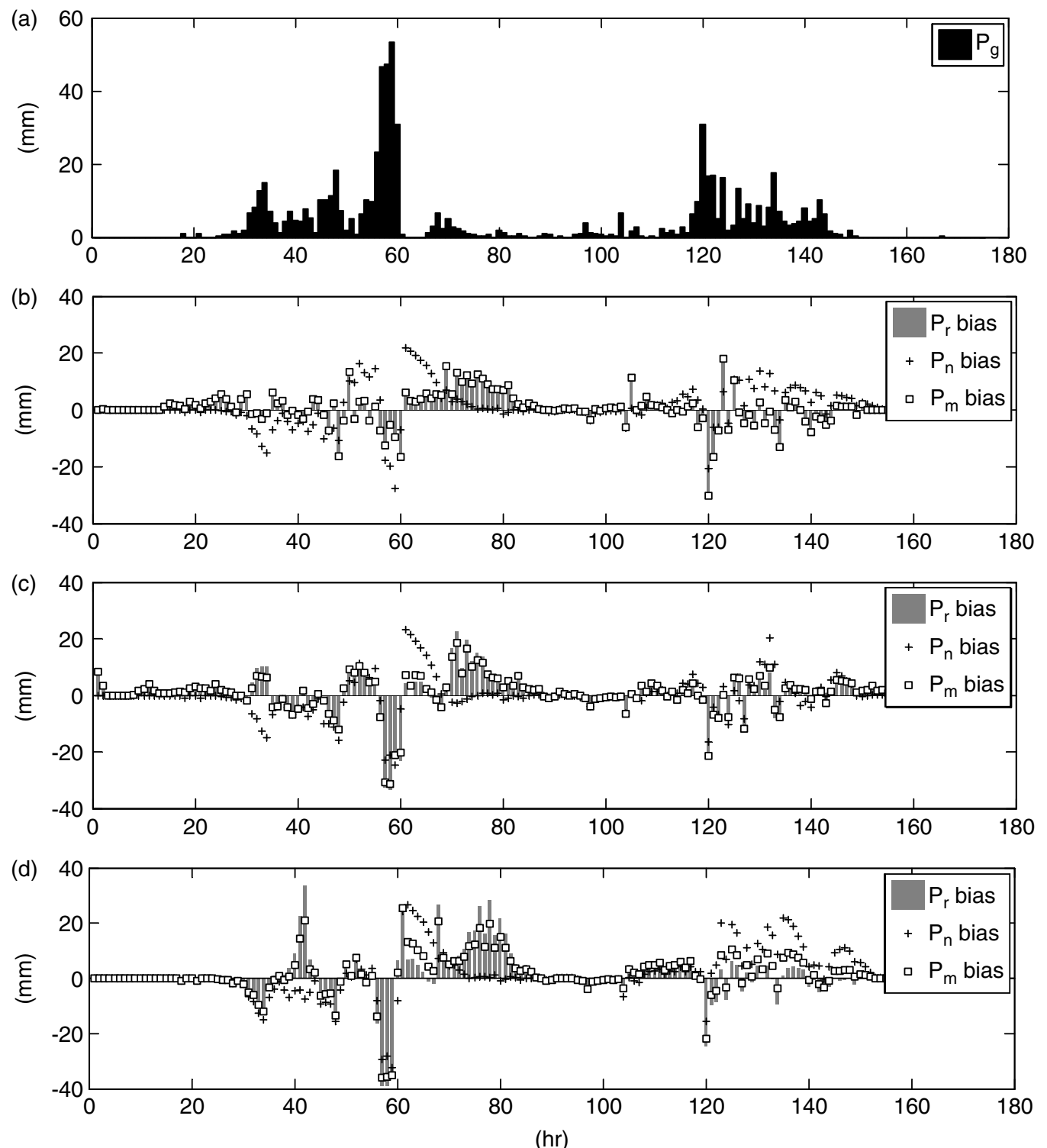

Figure 6. (a) The rainfall pattern obtained from gauge measurements and the forecasted bias for lead time (b) 1, (c) 3 , and (d) $6 \mathrm{~h}$

bias calculated from three QPF sequences for lead time 1,3 , and $6 \mathrm{~h}$.

Table 4 shows the contribution of $P_{r}$ on merged precipitation $\left(P_{m}\right)$ and the corresponding improvement $\left(S S_{1}\right)$ for 1-6-h precipitation predictions. The statistical error measurements of the reference model $\left(E_{R M}\right)$ can be referred to RMSE, which was calculated from present gauge measurements. Basically, the value of $\theta_{k}$ was gradually 
Table IV. Contribution of $P_{n}$ and $P_{r}$ on $P_{m}$ and the corresponding improvement

\begin{tabular}{lcccr}
\hline Lead time & $\theta_{k}$ & \multicolumn{3}{c}{$S S_{1}(\%)$} \\
\cline { 3 - 5 } & & $P_{n}$ & $P_{r}$ & $P_{m}$ \\
\hline 1 & 0.98 & -33 & -9.3 & $-8 \cdot 3$ \\
2 & 0.92 & $5 \cdot 6$ & 10.5 & $13 \cdot 6$ \\
3 & 0.84 & 21.5 & 23.0 & 29.3 \\
4 & 0.74 & $30 \cdot 0$ & 27.6 & 34.0 \\
5 & 0.72 & $30 \cdot 1$ & 26.2 & $34 \cdot 1$ \\
6 & 0.69 & $25 \cdot 8$ & 17.5 & 28.4 \\
\hline
\end{tabular}

reduced, which means more contributions were made from $P_{n}$ as the forecast lead time increased. It appears all methods have significantly better performance than the gauge measurements, except for a one-hour-ahead prediction with negative $S S_{1}$, which means no improvement can be made in one-hour-ahead prediction. Although the optimal merging parameter at a lead time of $2 \mathrm{~h}$ shows that the $P_{n}$ has limited contribution $(8 \%)$ to the merging procedure, the $P_{m}$ still has an improvement of $13.6 \%$ which is superior to $P_{n}(5 \cdot 6 \%)$ and $P_{r}(10.5 \%)$. For 3-6-h predictions, the contribution of $P_{n}$ on merging procedure is efficiently increased. Clearly, Table 4 displays quantitative values to facilitate a comparison of different models. These results indicate again that the developed model provides efficient precipitation forecasts for lead time up to $6 \mathrm{~h}$ and the NWP precipitation forecasts provide relative effectiveness to the merged products.

\section{Results of multistep ahead of flood forecasting}

The testing statistics of 1-6-h flood forecasts, in terms of CC, MAE, CE, and RMSE, based on three sets of inputs, denoted as $\mathrm{RNN}_{\mathrm{G}}, \mathrm{RNN}_{\mathrm{M}}$, and $\mathrm{RNN}_{\mathrm{I}}$, are shown in Table 5. Inspection of the comprehensive comparison of the above models for 1-6-h flood forecasting reveals several results: first, whichever hydrologic model is used, the efficiency of model output is fairly good for one-hour-ahead forecasting. This result indicates that the RNN has a potential advantage to accurately forecast the nonlinear and dynamic relationship between rainfall and runoff processes. Secondly, for the $\mathrm{RNN}_{\mathrm{I}}$ model, all the four statistics indicate that the hydrologic model can provide very satisfactory forecasts of up to $6 \mathrm{~h}$ if perfect precipitation predictions are available. However, it appears from Table 5 that, even with perfect precipitation predictions, the efficiency of hydrologic model responses does not change dramatically for oneto three-hour-ahead forecasting. On the contrary, the model efficiency is particularly improved for lead times of 4-6 h, suggesting that the hydrologic model in this study watershed is not sensitive to predicted precipitation for short-term (1-3 h) flood forecasting. Performance produced from $\mathrm{RNN}_{\mathrm{I}}$ strongly demonstrates that the runoff input supplied most of the information for 1-3-h flood forecasts.

Thirdly, the performance shown in Table 5 definitely indicates that the $\mathrm{RNN}_{\mathrm{M}}$ model efficiency is significantly better than the conventional case $\left(\mathrm{RNN}_{\mathrm{G}}\right)$. The result implies that it would be beneficial to use merged precipitation for accurate multistep ahead of flood forecasting. To assess the accuracy and efficiency of model prediction specifically, the $E T_{P}$ and $E Q_{P}$ statistics estimated from three models in testing phase are listed in Table 6 . The results indicate that forecasts generated by the $\mathrm{RNN}_{\mathrm{G}}$ model resulted in poor $E T_{P}$ and $E Q_{P}$ as forecast lead time increased. By comparing the $E Q_{P}$ index, similar results are obtained from all models except for the $\mathrm{RNN}_{\mathrm{G}}$ model

Table VI. Evaluation of the timing and peak flow prediction in testing phase

\begin{tabular}{|c|c|c|c|c|c|c|}
\hline \multirow{2}{*}{$\begin{array}{l}\text { Lead } \\
\text { time }\end{array}$} & \multicolumn{3}{|c|}{$E T_{P}(\mathrm{hr})$} & \multicolumn{3}{|c|}{$E Q_{P}(\%)$} \\
\hline & $\mathrm{RNN}_{\mathrm{G}}$ & $\mathrm{RNN}_{\mathrm{M}}$ & $\mathrm{RNN}_{\mathrm{I}}$ & $\mathrm{RNN}_{\mathrm{G}}$ & $\mathrm{RNN}_{\mathrm{M}}$ & $\mathrm{RNN}_{\mathrm{I}}$ \\
\hline 1 & 0 & 0 & 0 & $-7 \cdot 2$ & $0 \cdot 3$ & $-1 \cdot 7$ \\
\hline 2 & 1 & 0 & 0 & -18.4 & $-3 \cdot 7$ & 2.7 \\
\hline 3 & 2 & 1 & 1 & $-25 \cdot 8$ & $-11 \cdot 0$ & $7 \cdot 4$ \\
\hline 4 & 3 & 2 & 1 & $-32 \cdot 5$ & -24.4 & -24.8 \\
\hline 5 & 4 & 2 & 1 & $-37 \cdot 8$ & $-31 \cdot 8$ & $-28 \cdot 8$ \\
\hline 6 & 5 & 3 & 1 & $-43 \cdot 6$ & -39.9 & $-35 \cdot 4$ \\
\hline
\end{tabular}

Table V. Statistical performance of three RNN models

\begin{tabular}{|c|c|c|c|c|c|c|c|}
\hline \multirow{2}{*}{ Statistics } & \multirow{2}{*}{ Models } & \multicolumn{6}{|c|}{ Lead time } \\
\hline & & 1 & 2 & 3 & 4 & 5 & 6 \\
\hline \multirow[t]{3}{*}{$\mathrm{CC}$} & $\mathrm{RNN}_{\mathrm{G}}$ & 0.985 & 0.909 & 0.806 & 0.645 & 0.506 & 0.470 \\
\hline & $\mathrm{RNN}_{\mathrm{M}}$ & 0.990 & 0.944 & 0.897 & 0.862 & 0.825 & 0.679 \\
\hline & $\mathrm{RNN}_{\mathrm{I}}$ & 0.989 & $0 \cdot 951$ & 0.926 & $0 \cdot 910$ & 0.908 & $0 \cdot 900$ \\
\hline \multirow{3}{*}{ MAE } & $\mathrm{RNN}_{\mathrm{G}}$ & $17 \cdot 9$ & $34 \cdot 1$ & $52 \cdot 6$ & $65 \cdot 5$ & $70 \cdot 5$ & $73 \cdot 7$ \\
\hline & $\mathrm{RNN}_{\mathrm{M}}$ & $15 \cdot 7$ & $30 \cdot 4$ & $45 \cdot 7$ & $50 \cdot 9$ & $61 \cdot 6$ & $78 \cdot 3$ \\
\hline & $\mathrm{RNN}_{\mathrm{I}}$ & $11 \cdot 7$ & $24 \cdot 6$ & $35 \cdot 6$ & $40 \cdot 5$ & $46 \cdot 0$ & $54 \cdot 8$ \\
\hline \multirow[t]{3}{*}{$\mathrm{CE}$} & $\mathrm{RNN}_{\mathrm{G}}$ & 0.968 & $0 \cdot 812$ & 0.646 & 0.417 & $0 \cdot 236$ & $0 \cdot 211$ \\
\hline & $\mathrm{RNN}_{\mathrm{M}}$ & 0.979 & 0.890 & 0.803 & 0.736 & 0.676 & 0.462 \\
\hline & $\mathrm{RNN}_{\mathrm{I}}$ & 0.978 & 0.905 & 0.857 & $0 \cdot 818$ & 0.804 & $0 \cdot 768$ \\
\hline \multirow[t]{3}{*}{ RMSE } & $\mathrm{RNN}_{\mathrm{G}}$ & 48 & 117 & 160 & 206 & 236 & 240 \\
\hline & $\mathrm{RNN}_{\mathrm{M}}$ & 39 & 89 & 120 & 138 & 153 & 198 \\
\hline & $\mathrm{RNN}_{\mathrm{I}}$ & 40 & 83 & 102 & 115 & 119 & 130 \\
\hline
\end{tabular}



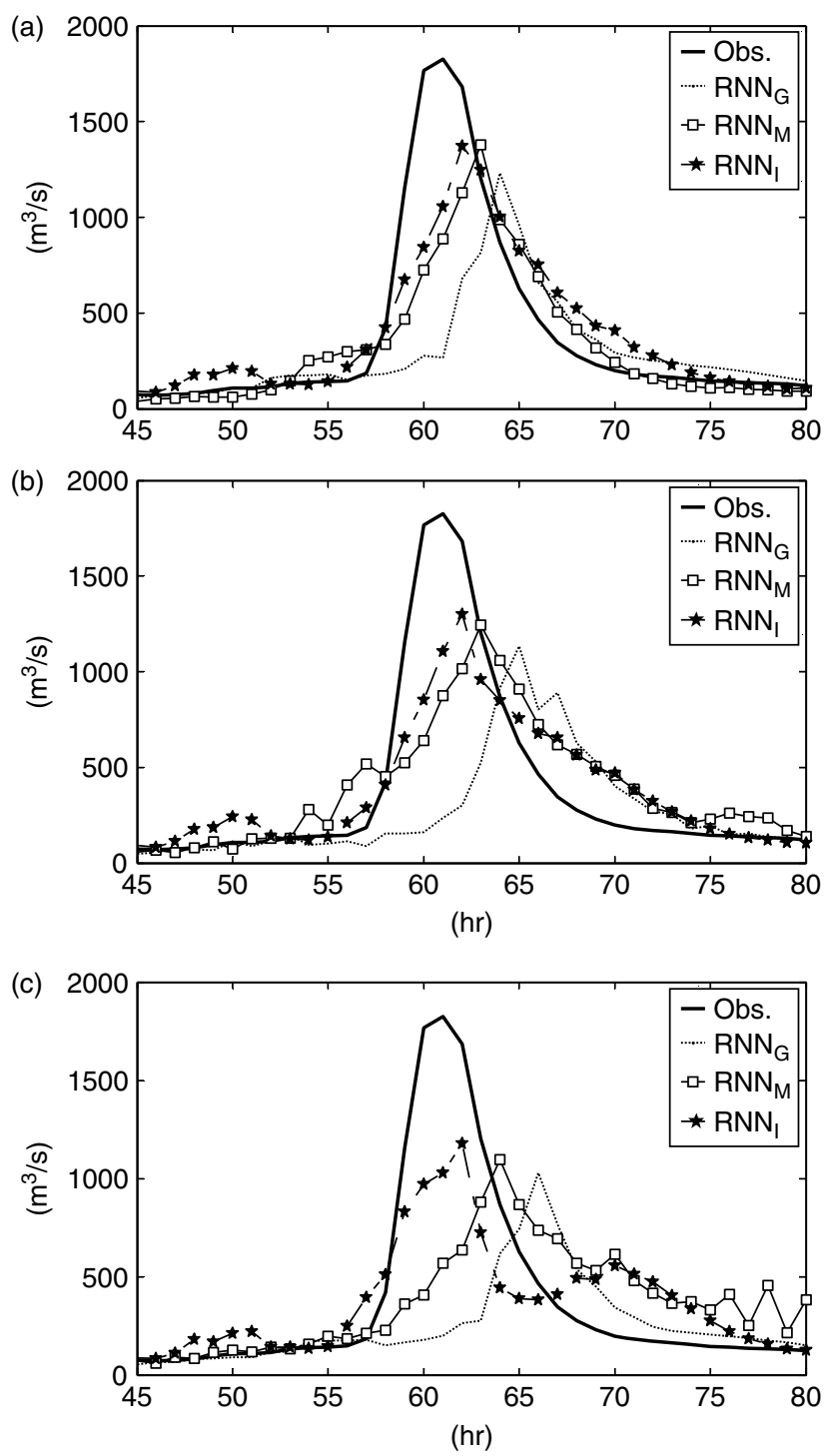

Figure 7. Observations versus forecasts for lead time of (a) 4, (b) 5, and (c) $6 \mathrm{~h}$

with slight underestimation of the peak flow. Moreover, both $\mathrm{RNN}_{\mathrm{M}}$ and $\mathrm{RNN}_{\mathrm{I}}$ models produced the same performance of $E T_{P}$ statistics for 1-3-h predictions. For 4-6-h predictions, the $\mathrm{RNN}_{\mathrm{I}}$ model maintained the forecasted timing very well $\left(E T_{P}=1\right)$; whereas the time-lag of $\mathrm{RNN}_{\mathrm{M}}$ for 4-6-h prediction are 2, 2, and $3 \mathrm{~h}$ which were better than that of $\mathrm{RNN}_{\mathrm{G}}$ model $(3,4$, and $5 \mathrm{~h})$. The result suggests that the time-lag problem, particularly for 4-6-h predictions, can be improved by using precipitation forecasts as model inputs. Figure 7 clearly shows the difference of timing of the forecasted peak flow for 4-6-h flood forecasting. Overall, the results shown in Figure 7 and Tables 5 and 6 strongly suggest that the $\mathrm{RNN}_{\mathrm{M}}$ model provided an accurate and stable 1-6-h flood forecasting as compared with the $\mathrm{RNN}_{\mathrm{G}}$, model and have substantially improved RMSE, as well as other statistics, such as $\mathrm{CC}, \mathrm{CE}, \mathrm{MAE}, E T_{P}$, and $E Q_{P}$.

Finally, the major issue here is to assess the impact of the merged precipitation product that is combined from two predicted precipitation sources on the multistep ahead of flood forecast accuracy. Table 7 shows the results of $\mathrm{RNN}_{\mathrm{M}}$ model in terms of $S S_{2}$ as compared with the $\mathrm{RNN}_{\mathrm{G}}$ model. In general, the $\mathrm{RNN}_{\mathrm{M}}$ model clearly shows excellent performance for 1-6-h flood forecasting with positive $S S_{2}$. Moreover, the improvement gradually increases with $S S_{2}$ varying from $18 \cdot 8-35 \cdot 2 \%$ for $1-5$ $\mathrm{h}$ flood forecasting. Even though the improvement of $\mathrm{RNN}_{\mathrm{M}}$ in a lead time of $6 \mathrm{~h}$ is slightly low, the forecasts remain much more accurate $\left(S S_{2}=17.5 \%\right)$ as compared with $\mathrm{RNN}_{\mathrm{G}}$ forecasts. This is because the model forecasts in the vicinity of peak flood are somewhat underestimated. On the basis of the above comprehensive results, the study suggests that improved 1-6-h flood forecasting can be appropriately generated via accurate QPF information which is obtained by merging two QPF products.

\section{CONCLUSIONS}

The major purpose of this study was to effectively construct an accurate 1-6-h flood forecasting during typhoon periods since floods are one of the major natural hazards that frequently cause societal and economical damages in Taiwan. For better flood mitigation, there is a necessity to conduct interdisciplinary research, such as in hydrology, meteorology, and hydrometeorology. To achieve the goal, we presented a preliminary investigation on the applications of hydrometeorological information for (1) 1-6-h precipitation forecasting, and (2) 1-6-h flood forecasting.

For 1-6 hQPF, a novel technique that utilizes a 3-D structure of radar echo, together with the meteorological variables generated from NWP model was first developed by using aBPNN; and then a more accurate precipitation product was merged by linearly combining the NWP precipitation forecasts and radar/NWP multisource precipitation forecasts. To find the effective weighted merging parameters, a RNN was used for minimizing the predictive error. On the basis of the comparative results, the merging procedure demonstrates its ability to produce more accurate and robust precipitation information than that of gauge measurement. Besides, the precision of merged precipitation is higher than radar/NWP multisource precipitation forecasts, which indicates that the NWP precipitation forecasts do provide relative effectiveness for the merging procedure, particularly for a forecast lead time of 4-6 h. Overall, the merged precipitation products performed well and captured the main tendency of rainfall patterns. The results also demonstrate

Table VII. The $S S_{2}$ values of $\mathrm{RNN}_{\mathrm{M}}$ model for $1-6$-h flood forecasting

\begin{tabular}{ccccccc}
\hline & \multicolumn{6}{c}{ Lead time } \\
\cline { 2 - 7 } & 1 & 2 & 3 & 4 & 5 & 6 \\
\hline $\mathrm{SS}_{2}(\%)$ & $18 \cdot 8$ & 23.9 & $25 \cdot 0$ & $33 \cdot 0$ & $35 \cdot 2$ & $17 \cdot 5$ \\
\hline
\end{tabular}


that the radar-derived and NWP precipitation forecasts can be integrated adequately by the merging procedure.

Subsequently, several 1-6-h flood forecasting models were then investigated from different scenarios. Preliminary research explored the effectiveness of merged precipitation and its potential improvement in 1-6-h flood forecasting. The results indicate that the merging procedure used in this study can efficiently combine the information from both precipitation products and increase the applicability of 1-6-h flood forecasting during typhoon periods. The evaluation of 1-6-h flood forecasting schemes strongly shows that the $\mathrm{RNN}_{\mathrm{M}}$ model provided accurate and stable flood forecasts in comparison with conventional cases and significantly improved the peak flow forecasts and the time-lag problem. Another important finding is that the hydrologic responses do not seem to be sensitive to precipitation predictions but runoff information at the first few lead times, whereas the model forecasts are highly dependent on QPF information for longer lead times. The results obtained in this study also show that the hydrological model still has the possibility of improving the accuracy of flood forecasts if better precipitation predictions can be provided in the future.

\section{REFERENCES}

Chang FJ, Chang YT. 2006. Adaptive neuro-fuzzy inference system for prediction of water level in reservoir. Advances in Water Resources 29: $1-10$.

Chang FJ, Chiang YM, Chang LC. 2007. Multi-step-ahead flood forecasting by neural networks. Hydrological Sciences Journal 52: $114-130$.

Chang LC, Chang FJ, Chiang YM. 2004. A two-step ahead recurrent neural network for stream-flow forecasting. Hydrological Processes 18: $81-92$.

Chattopadhyay S, Chattopadhyay G. 2008. Comparative study among different neural net learning algorithms applied to rainfall time series. Meteorological Applications 15: 273-280.

Chaves P, Kojiri T. 2007. Deriving reservoir operational strategies considering water quantity and quality objectives by stochastic fuzzy neural networks. Advances in Water Resources 30: 1329-1341.

Chiang YM, Chang LC, Chang FJ. 2004. Comparison of staticfeedforward and dynamic-feedback neural networks for rainfall-runoff modeling. Journal of Hydrology 290: 297-311.

Chiang YM, Chang FJ, Jou BJD, Lin PF. 2007a. Dynamic artificial neural network for real-time precipitation estimation and forecasting from radar observations. Journal of Hydrology 334: 250-261.

Chiang YM, Hsu KL, Chang FJ, Hong Y, Sorooshian S. 2007b. Merging multiple precipitation sources for flash flood forecasting. Journal of Hydrology 340: 183-196.

Droegemeier KK, Smith JD, Businger S, Doswell C, Doyle J, Duffy C, Foufoula-Georgiou E, Graziano T, James LD, Krajewski V, LeMone M, Lettenmaier D, Mass C, Pielke R, Ray P, Rutledge S, Schaake J, Zipser E. 2000. Hydrological aspects of weather prediction and flood warnings. Bulletin of the American Meteorological Society 81: $2665-2680$.
Firat M, Güngör M. 2008. Hydrological time-series modelling using an adaptive neuro-fuzzyinference system. Hydrological Processes 22: 2122-2132.

Ganguly AR, Bras RL. 2003. Distributed quantitative precipitation forecasting Using information from radar and numerical weather prediction models. Journal of Hydrometeorology 4: 1168-1180.

Giustolisi O, Laucelli D. 2005. Improving generalization of artificial neural networks in rainfall-runoff modeling. Hydrological Sciences Journal 50: 439-457.

Grecu M, Krajewski WF. 2000. Rainfall forecasting using variational assimilation of radar data in numerical cloud models. Advances in Water Resources 24: 213-224.

Grimes DIF, Coppola E, Verdecchia M, Visconti G. 2003. A neural network approach to real-time rainfall estimation for Africa using satellite data. Journal of Hydrometeorology 4: 1119-1133.

Golding BW. 2000. Quantitative precipitation forecasting in the UK Journal of Hydrology 239: 286-305.

Ham FM, Kostanic I. 2001. Principles of Neurocomputing for Science and Engineering. McGraw-Hill: New York.

Hsu KL, Gupta HV, Sorooshian S. 1995. Artificial neural network modeling of the rainfall-runoff process. Water Resources Research 31: 2517-2530.

Khan MS, Coulibaly P. 2006. Bayesian neural network for rainfallrunoff modeling. Water Resources Research 42(7): W07409. DOI:10.1029/2005WR003971.

Kidd C, Kniveton DR, Todd MC, Bellerby TJ. 2003. Satellite rainfall estimation using combined passive microwave and infrared algorithms. Journal of Hydrometeorology 4: 1088-1104.

Kisi O. 2007. Streamflow forecasting using different artificial neural network algorithms. Journal of Hydrologic Engineering 12: 532-539.

Kuligowski RJ, Barros A. 1998. Localized precipitation forecasts from a numerical weather prediction model using artificial neural networks. Weather and Forecasting 13: 1194-1204.

Luk KC, Ball JE, Sharma A. 2000. A study of optimal model lag and spatial inputs to artificial neural network for rainfall forecasting. Journal of Hydrology 227: 56-65.

Meneguzzo F, Pasqui M, Menduni G, Messeri G, Gozzini B, Grifoni D, Rossi M, Maracchi G. 2004. Sensitivity of meteorological highresolution numerical simulations of the biggest floods occurred over the Arno river basin, Italy, in the 20th century. Journal of Hydrology 288: $37-56$.

Minns AW, Hall MJ. 1996. Artificial neural networks as rainfall-runoff models. Hydrological Sciences Journal 41: 399-417.

Mittermaier MP. 2008. Introducing uncertainty of radar-rainfall estimates to the verification of mesoscale model precipitation forecasts. Natural Hazards and Earth System Sciences 8: 445-460.

Rogers RR, Yau MK. 1989. A Short Course in Cloud Physics. Pergamon Press; 293.

Sivakumar B, Jayawardena AW, Fernando TMKG. 2002. River flow forecasting: use of phase-space reconstruction and artificial neural networks approaches. Journal of Hydrology 265: 225-245.

Sokol Z. 2006. Nowcasting of 1-h precipitation using radar and NWP data. Journal of Hydrology 328: 200-211.

Valverde Ramirez MC, de Campos Velho HF, Ferreira NJ. 2005. Artificial neural network technique for rainfall forecasting applied to the Sao Paulo region. Journal of Hydrology 301: 146-162.

Xiao R, Chandrasekar V. 1997. Development of a neural network based algorithm for rainfall estimation from radar observations. IEEE Transactions on Geoscience and Remote Sensing 35: 160-171.

Yussouf N, Stensrud DJ. 2008. Reliable probabilistic quantitative precipitation forecasts from a short-range ensemble forecasting system during the 2005/06 cool season. Monthly Weather Review 136: $2157-2172$. 